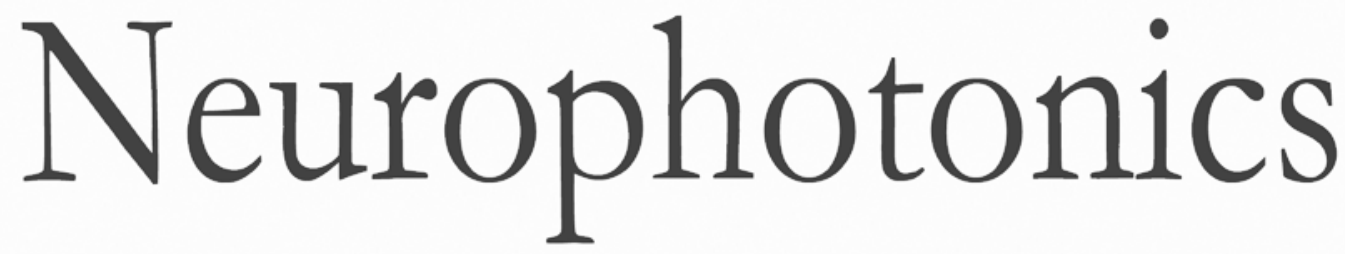

\title{
Neural activation within the prefrontal cortices during the goal- directed motor actions of children with hemiplegic cerebral palsy
}

Swati M. Surkar

Rashelle M. Hoffman

Regina Harbourne

Max J. Kurz 


\title{
Neural activation within the prefrontal cortices during the goal-directed motor actions of children with hemiplegic cerebral palsy
}

\author{
Swati M. Surkar, ${ }^{\mathrm{a}}$ Rashelle M. Hoffman, ${ }^{\mathrm{a}}$ Regina Harbourne, ${ }^{\mathrm{b}}$ and Max J. Kurz ${ }^{\mathrm{a}, *}$ \\ aUniversity of Nebraska Medical Center, Munroe Meyer Institute of Genetics and Rehabilitation, Department of Physical Therapy, Omaha, \\ Nebraska, United States \\ ${ }^{\mathrm{b}}$ Duquesne University, Department of Physical Therapy, Pittsburgh, Pennsylvania, United States
}

\begin{abstract}
The primary aim of the study was to explore the prefrontal cortical (PFC) activation while performing a shape-matching motor task in children with hemiplegic cerebral palsy (HCP) as compared with typically developing (TD) children. Fifteen TD children (age $=5.9 \pm 1.1$ years) and 12 children with $\mathrm{HCP}$ (age $=$ $6.8 \pm 2.9$ years) were included. We assessed the PFC activation while performing an ecologically valid upper extremity shape-matching task of different complexities by measuring the concentration of oxygenated hemoglobin $(\mathrm{HbO})$ using functional near-infrared spectroscopy. The motor task performance was assessed by quantifying the average number of shapes matched, reaction time (RT), task errors, nine-hole peg test (NHPT), and the box and block test (BBT). Overall, there was a systematic increase in the $\mathrm{HbO}$ in the PFC across the shape-matching complexity conditions. Our results also revealed that the children with HCP had an increased amount of PFC activation while performing all of the shape-matching tasks. The increased PFC activation paralleled the differences in the number of shapes matched, RT, task errors, NHPT, and BBT. The atypical motor actions seen in children with HCP may be partially related to the greater cognitive demands placed on the PFC. @ 2018 Society of Photo-Optical Instrumentation Engineers (SPIE) [DOI: 10.1117/1.NPh.5.1.011021]
\end{abstract}

Keywords: action planning; cognition; motor performance; movement dysfunction.

Paper 17059SSRRR received Mar. 31, 2017; accepted for publication Feb. 8, 2018; published online Mar. $10,2018$.

\section{Introduction}

The unilateral sensorimotor dysfunction in children with hemiplegic cerebral palsy $(\mathrm{HCP})$ can result in the loss of upper extremity motor control, which affects activities of daily living and restricts the child's participation in educational, leisure, and vocational roles. ${ }^{1}$ Until recently, action execution problems residing in the musculoskeletal machinery were considered as primarily responsible for activity limitations in children with $\mathrm{HCP}^{2}$ However, emerging evidence suggests that the activity limitations and action performance problems seen in these children are not solely an action execution disorder but might also be due to deficits in planning of the goal-directed actions. ${ }^{3,4}$

Action planning is the ability to predict the future state of the motor system and is integral for the control of skilled movements. ${ }^{3,5}$ According to the planning-control model, action planning has two main components: (a) premovement planning and (b) online monitoring and correction of the movement to achieve the goal state. ${ }^{6}$ Premovement planning involves processes, such as goal determination, target identification, selection, analysis of object affordances, timing, and computation of the target size, shape, orientation, and position relative to the body. ${ }^{7}$ Online control involves visual and proprioceptive feedback to monitor movement and minimize spatial errors. ${ }^{7}$ Behavioral studies reveal that children with HCP have a deficit at the action planning level. ${ }^{8-12}$ This notion is based on the observation that children with HCP have task failures, ${ }^{8}$ uncomfortable grip selection, loss of the end-state-comfort effect, ${ }^{8,9}$ and difficulty in

*Address all correspondence to: Max J. Kurz, E-mail: mkurz@unmc.edu anticipating grip forces. ${ }^{10}$ They also take a longer time to plan sequential movements ${ }^{11}$ and lack fluid movements. ${ }^{12}$ Consequently, the presence of an action-planning deficit likely limits the ability to successfully execute movements. Although these behavioral observations may be accurate, these observations alone cannot determine whether the source of aberrant movements stems from impaired musculoskeletal machinery (i.e., spasticity, muscle weakness or lack of selective control, joint torsions, and contractures), faulty cognitive processes (i.e., attention, memory, and information processing), or a combination of both.

Over the past decade, the neuroimaging of movement-related brain activity has substantially advanced our understanding of how adults and children plan and produce goal-directed movements. ${ }^{13-21}$ These studies have shown that the production of goal-directed movements involves the activation of a distributed network that includes the primary sensorimotor cortices, secondary somatosensory area, parietal cortices, supplementary motor area, basal ganglia, thalamus, and cerebellum. In addition, such studies have also highlighted that the prefrontal cortex (PFC) also plays a critical role in planning and monitoring of the evolving actions. ${ }^{5,22}$ The PFC has extensive connections with the premotor and sensorimotor cortex..$^{23}$ Although it is well recognized that these brain areas are involved in the control of movement, the neurophysiology literature on children with HCP has predominantly focused on identifying the structural aberrations within the white matter volume and the fiber tracks that are related to aberrant actions. ${ }^{24-30}$ Specifically, these 
studies have used transcranial magnetic stimulation and diffusion tensor imaging to assess corticospinal tract integrity and focused on the relationship between the cortical organization and the hand function. ${ }^{24-28}$ The absence of response on the hemiplegic arm after stimulating contralateral hemisphere and disruption of corticospinal tract was found to be associated with poor motor hand function. ${ }^{29,30} \mathrm{~A}$ few studies that have evaluated the cortical activity of children with HCP have shown that the sensorimotor cortices can be hyperactivated and may involve compensatory networks when planning and executing goaldirected actions. ${ }^{31-34}$ However, these insights have been gained from the evaluation of simple motor actions (i.e., knee and hand movements) that do not involve higher order cognitive decisions and maintenance of goal relevant information. Potentially, the evaluation of more ecologically valid motor tasks may improve our understanding of how the central processing deficits impact the goal-directed motor actions seen in children with HCP.

In this exploratory investigation, we used functional nearinfrared spectroscopy (fNIRS) to measure the PFC activation as typically developing (TD) children and children with HCP performed a shape-matching motor task with their upper extremities. The shape-matching task encompasses (a) premovement planning, which involves various cognitive processes to make a decision of an appropriate shape match and to manipulate the different shapes and (b) online control of movement, which involves action of reaching, grasping, and orientating the shapes accurately. Our primary hypothesis was that, compared with the TD children, the children with HCP would show an increased amount of neural activity in the PFC due to greater utilization of cognitive resources that are required for planning and control of their actions. Our secondary hypothesis was that the deficits in action planning might impair the motor performance.

\section{Methods}

\subsection{Participants}

The Institutional Review Board of the University of Nebraska Medical Center (UNMC) approved the study, and we obtained parental consent and child assent to participate in this investigation. The participating children with HCP were recruited from the physical therapy clinic at UNMC and TD children were recruited through word of mouth. The physical therapists, who had access to the children's health records, prescreened the children based on the inclusion and exclusion criteria to qualify for the study. The exclusion criteria were the presence of frontal cortical lesions, cognitive impairments, visual deficits, musculoskeletal deformity of the hand and arm, such as any fixed contractures of hand or wrist, which limited the ability to grasp and manipulate objects, manual ability classification level (MACS) level I (handles objects easily and successfully) and V (does not handle objects and has severely limited ability to perform simple actions), and arm weakness due to neurological impairments such as brachial plexus injuries. Twelve children with HCP $($ age $=6.8 \pm 2.9$ years; males $=7)$ and 15 TD children $($ age $=5.8 \pm 1.1$ years; males $=8)$ fit the inclusion criteria and participated in this investigation. All children with HCP had a previously defined diagnosis of hemiplegia by a pediatric neurologist. Further details of the participating children are shown in Table 1.

\subsection{Clinical Assessments}

As an initial screening, we assessed manual ability of children with HCP using manual ability classification system (MACS). ${ }^{35}$ A certified assisting hand assessment (AHA) assessor also performed the AHA in children with HCP using a standardized, semistructured, 10 to 15 -min play session with toys requiring bimanual use of the arm. ${ }^{36,37}$ The session was video-recorded and later scored to determine bimanual coordination and assisting hand function of children with HCP. ${ }^{37}$ Lastly, we had the children performing the nine-hole peg test (NHPT) and the box and blocks test (BBT) to assess manual dexterity and speed. ${ }^{38,39}$ Moreover, the handedness for all children was determined using Edinburgh handedness inventory. ${ }^{40}$

\subsection{Functional Near-Infrared Spectroscopy Experimental Paradigm}

The task consisted of a sequential shape-matching task, which was developed for this study. This task consisted of three-complexity levels, such as easy, moderate, and difficult. The easy condition had the same shape types, the moderate condition had two different shape types, and the difficult condition had multiple shape types that were different from each other (Fig. 1). The three-complexity levels of the task were based on the intricacy of the shape identification, accurate selection, manipulation, and the type of grasp required based on the type, size, shape, and orientation of the shape. The children were asked to match the shapes with the corresponding template by selecting an appropriate shape and placing it accurately on a given template. The likelihood that the different complexity levels of the shape-matching task would elicit changes in the $\mathrm{HbO}$ concentration within the PFC was initially determined with a pilot study that we conducted with five TD children. The results of this preliminary analysis are shown in Table 2.

The shape-matching task was performed in a block paradigm, which consisted of a 30 -s rest period where the child sat still and a 30-s active period where the child matched the shapes. The children did not have a chance to visualize the shapes during the rest period. To avoid time for prior planning of the task, the assessor presented the template and shapes at time 0 . We allowed children to match as many shapes as they could within the 30 -s active period. Typically, the children could not match all 12 shapes in this time frame. However, in the rare instance, if the child matched all 12 shapes, the assessor removed the shapes from the template and the child continued matching the shapes on the same template until the $30 \mathrm{~s}$ of the active period was over. To avoid anticipation of the respective complexity levels, the conditions were randomized and each task condition was repeated four times. The children performed a total of 12 blocks of the shape-matching task (three shape complexity conditions $\times$ four repetitions of each condition) during the entire session with an alternating pattern of the $30 \mathrm{~s}$ active and rest periods. The total duration of the data collection was $12 \mathrm{~min}$. Children with HCP performed the task with the affected and the less affected arm, and TD children performed the task with the dominant and the nondominant hand. We chose to evaluate both arms to explore the global nature of cognitive processes required for the movement planning and control and to avoid the arm bias of the hemiplegic hand due to physical restrictions in performing the task in view of impairments in the affected arm. 
Table 1 Demographic details of the participating TD and children with HCP.

\begin{tabular}{|c|c|c|c|c|c|c|c|c|c|}
\hline \multicolumn{7}{|c|}{$\mathrm{HCP}$} & \multicolumn{3}{|c|}{ TD } \\
\hline & Gender & Age (years) & Side of hemiplegia & MACS level & AHA Score & Diagnosis & & Gender & Age (years) \\
\hline HCP 1 & M & 4.5 & L & IV & 07 & Perinatal stroke & TD 1 & M & 6.7 \\
\hline HCP 2 & M & 4.6 & $\mathrm{R}$ & II & 85 & Perinatal stroke & TD 2 & $\mathrm{~F}$ & 6.6 \\
\hline HCP 3 & M & 5.3 & $\mathrm{R}$ & IV & 12 & Perinatal stroke & TD 3 & $\mathrm{~F}$ & 4.1 \\
\hline HCP 4 & $\mathrm{~F}$ & 6.1 & L & III & 59 & Perinatal stroke & TD 4 & $M$ & 6.6 \\
\hline HCP 5 & $\mathrm{~F}$ & 6.1 & L & II & 87 & PVL & TD 5 & $\mathrm{~F}$ & 4.6 \\
\hline HCP 6 & M & 11.0 & L & III & 52 & Neonatal stroke & TD 6 & $\mathrm{~F}$ & 4.1 \\
\hline HCP 7 & M & 12.0 & $\mathrm{R}$ & III & 70 & Neonatal stroke & TD 7 & $M$ & 6.5 \\
\hline HCP 8 & $\mathrm{~F}$ & 11.0 & L & III & 72 & Neonatal stroke & TD 8 & $\mathrm{~F}$ & 7.5 \\
\hline HCP 9 & M & 5.0 & L & III & 64 & Schizencephaly & TD 9 & $\mathrm{~F}$ & 6.8 \\
\hline HCP 10 & $\mathrm{~F}$ & 4.1 & L & III & 58 & Perinatal stroke & TD 10 & M & 7.0 \\
\hline HCP 11 & $\mathrm{~F}$ & 4.8 & $\mathrm{R}$ & III & 58 & Perinatal stroke & TD 11 & M & 5.11 \\
\hline \multirow[t]{4}{*}{ HCP 12} & $M$ & 7.6 & L & III & 62 & Neonatal stroke & TD 12 & $M$ & 4.6 \\
\hline & & & & & & & TD 13 & $\mathrm{~F}$ & 6.11 \\
\hline & & & & & & & TD 14 & $\mathrm{~F}$ & 5.11 \\
\hline & & & & & & & TD 15 & $\mathrm{~F}$ & 6.0 \\
\hline
\end{tabular}

Note: PVL, periventricular leukomalacia; MACS, manual ability classification system; AHA, assisting hand assessment; M, male; F, female; L, left; and $R$, right.

Note: All TD children were right hand dominant.

(a)

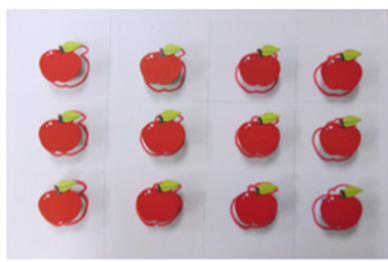

(b)

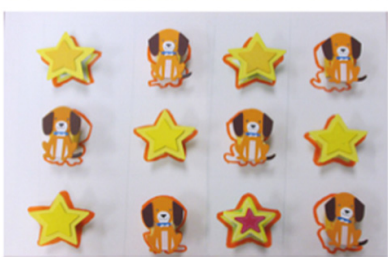

(c)

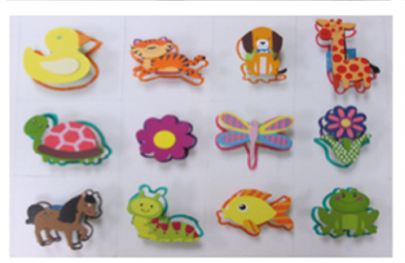

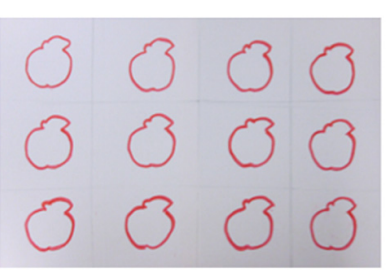
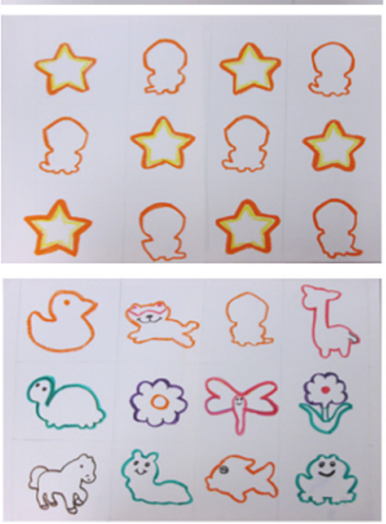

Fig. 1 Experimental shape-matching conditions. The shapes template was presented to the child just before beginning the task. (a) Easy: the easy condition had the same shape types, (b) moderate: the moderate condition had two different shape types, and (c) difficult: the difficult condition had multiple shape types that were different from each other. The children were asked to match the maximum number of shapes with the corresponding template for $30 \mathrm{~s}$. The conditions were randomized and each task condition was repeated four times.

\subsection{Functional Near-Infrared Spectroscopy Data Acquisition}

fNIRS is a neuroimaging technique that utilizes specific wavelengths of infrared light to approximate the absorption characteristics of oxygenated ( $\mathrm{HbO})$ and deoxygenated ( $\mathrm{HbR})$ hemoglobin within the underlying tissues ${ }^{41}$ Although the infrared light is partially absorbed by nonneural tissue, the relative change in the $\mathrm{HbO}$ or $\mathrm{HbR}$ is linked with the change in the underlying neural dynamics of the cortices. The fNIRS device consists of a series of photon emitters and detectors. The detectors measure the refracted light, which is used to quantify the amount of $\mathrm{HbO}$ and $\mathrm{HbR}$ changes in local neural tissues. A greater concentration of $\mathrm{HbO}$ corresponds to a heightened amount of activity in the underlying neural tissues. ${ }^{41}$ fNIRS is a noninvasive, safe, and portable method for assessing cortical activity. It is robust with respect to body movements and is not performed in a constrained environment like what is seen in a functional magnetic resonance imaging (fMRI) bore. Additionally, fNIRS is quiet (no operating sound) and can be used to assess the ecologically valid motor tasks. Because of all these advantages, fNIRS was used to address the research questions put forth in this investigation.

For this experiment, we used a continuous wave fNIRS system (fNIR Devices LLC, Potomac, Maryland) that utilized two different wavelengths ( 730 and $850 \mathrm{~nm}$ ) to measure the concentration of $\mathrm{HbO}$ and $\mathrm{HbR}$ based on the modified Beer-Lambert law. ${ }^{42}$ The fNIRS system (Fig. 2) was composed of three 
Table 2 Pilot data that were performed with a cohort of TD children $(N=5$; age $=5.56 \pm 0.63$ years; males $=4)$ that were right hand dominant. The children completed the shape-matching task that was used in this investigation. The maximum $\mathrm{HbO}$ concentration ( $\mu$ mol) seen during the active period was calculated as a preliminary measure of the neural activity in the prefrontal cortices. Trends in the data suggested that the maximum $\mathrm{HbO}$ was highest for difficult condition followed by moderate and easy conditions. $R=$ right arm and $L=$ left arm.

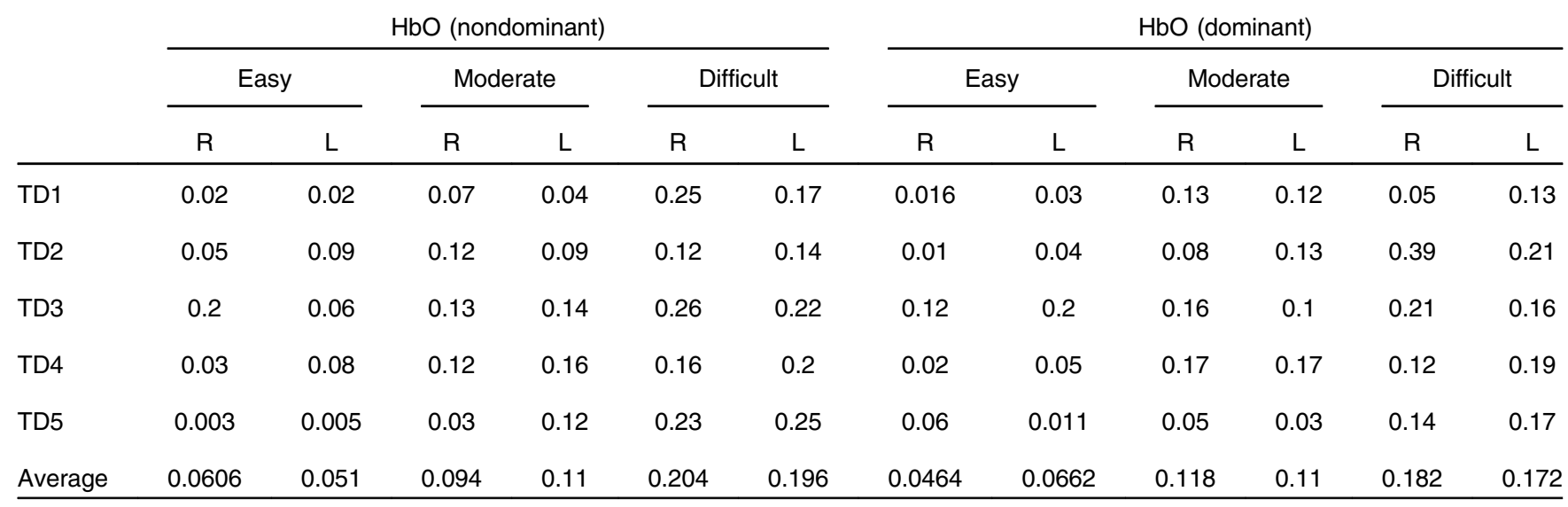

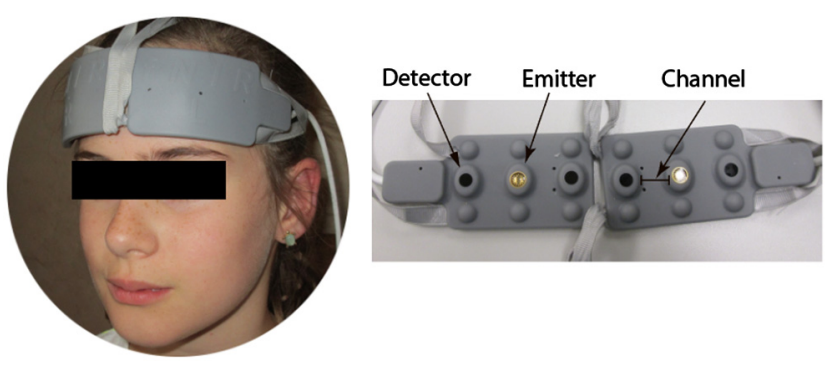

Fig. 2 Depiction of the placement of the fNIRS system on an exemplary child. The system consists of a flexible sensor pad that is positioned on the forehead based on the 10 to 20 EEG system. The flexible sensor pad contains the emitters and photo-detectors. This arrangement results in two measurement channels per hemisphere.

components: a flexible head piece, which secures the emitters and detectors in a fixed position to allow for fast placement of the sensor pad on the forehead; a control box for hardware; and a computer that runs the data acquisition. The positioning of two light sources and two detectors on the sensor pad yielded a total of four measurement channels. According to 10 to $20 \mathrm{EEG}$ system, the optodes were positioned lateral to the Fpz on the left and right sides of the forehead. The sensors had 2-Hz sampling rate and measured the hemodynamic change in $\sim 5$ to $10 \mathrm{~mm}$ of the outermost cortical tissue. ${ }^{43-45}$ All optodes were connected to fiber optic cables that allowed the transmission of infrared light to the fNIRS system. We used cognitive optical brain imaging studio software for data acquisition and visualization (fNIR Devices LLC, Potomac, Maryland).

\subsection{Functional Near-Infrared Spectroscopy Data Processing and Analysis}

We used the fNIRSoft software (v 3.1; fNIR Devices LLC, Potomac, Maryland) to calculate the optical density of the raw light intensity signals, and the implementation of a modified Beer-Lambert law to determine the changes in the $\mathrm{HbO}$ concentration. ${ }^{46}$ Waveforms that were saturated or had motion artifacts were visually identified and excluded from the analysis. A fourth-order digital filter was applied to low- and high-pass filter of the $\mathrm{HbO}$ time series with 0.1 and $10 \mathrm{~Hz}$ cutoffs, respectively. These filter settings were applied to reduce the potential effects of the physiological noise (e.g., equipment noise, respiration, and cardiac cycle effects) that often accompanies the measured cortical hemodynamics. ${ }^{47-49}$ The epochs of each trial were $60 \mathrm{~s}$ in duration $(-30$ to $+30 \mathrm{~s})$, with the presentation of the shape-matching task defined as $0.0 \mathrm{~s}$. The HbO hemodynamic waveforms for each channel were corrected based on the average $\mathrm{HbO}$ seen in the baseline period ( -10 to $0 \mathrm{~s}$ ), and the four trials performed in each condition were averaged to create the time course of the concentration of the $\mathrm{HbO}$ for the right and left PFCs, respectively. Subsequently, average $\mathrm{HbO}$ across the active period was calculated as a proxy measure of the neural activity in the PFC. We used the average $\mathrm{HbO}$ as a marker for regional brain activation since previous study findings have shown that $\mathrm{HbO}$ is more sensitive to neural changes than $\mathrm{HbR}^{50}$

\subsection{Behavioral Data Analysis}

The video-recorded behavioral data for the shape-matching task were used for the analysis of the motor task performance. The number of accurately matched shapes was quantified across each trial, and the average performance across the four trials for each condition was used as an outcome variable. We also assessed an average number of errors in matching the shapes across all trials. A wrong match of the shape on the corresponding template, use of the arm other than the testing arm, and inaccurate orientation of the shapes were considered as errors. In addition, we assessed reaction time (RT), which was determined as time to initiate the hand movement after the shape-matching task was presented. RT for the first shape in each trial was assessed, and average RT across all trials was considered for the final analysis. Performance of NHPT and BBT was also video-recorded and scores were calculated by quantifying the time required to complete the NHPT and number of blocks completed during the BBT.

\section{Statistical Analysis}

Separate mixed model analysis of variance (ANOVA) (group $\times$ side $\times$ arm $\times$ task-conditions) with group (TD and HCP) as the 
between-subject factor, and side (right and left PFC), arm (dominant/less affected, nondominant/affected), and task conditions (easy, moderate, and difficult) as the within-subject factors were used to determine if there were significant differences in $\mathrm{HbO}$, task performance, RT, and task errors. Separate $2 \times 2$ mixed ANOVAs with group (TD and HCP) as the between-subject factor, and arm (dominant/less affected and nondominant/affected) as the within-subject factors were used to determine if there were significant differences in the NHPT and BBT. Significant interaction effects were followed up with a least squared difference posthoc analysis. Cohen's $d$ was additionally calculated to determine the effect sizes of the results. The calculated effect sizes are interpreted as: $0.2-$ small, 0.5 -medium, and $>0.8$ as large ${ }^{51}$ Lastly, Pearson product moment correlations were conducted between the HbOs seen for the respective task complexity levels and the respective behavioral outcome variables collected from the entire cohort of children. All statistical analysis was performed using SPSS (Version 22.0; IBM Corporation, Armonk, New York) and $P$ values equal to or less than the corrected 0.01 alpha levels were considered to be significant. Results in the text and graphs are presented as a mean \pm standard error of the mean.

\section{Results}

\subsection{Prefrontal Cortical Activation}

The arm-specific average $\mathrm{HbO}$ concentrations for children with HCP and TD children during the respective conditions are shown in Fig. 3. The overall impression is that the average $\mathrm{HbO}$ concentration in the PFC is greater for the children with HCP across the respective conditions. The primary statistical results that are presented in the following sections are shown in Table 3.

There was a significant condition main effect $(P=0.001$; $d=1.31)$. Posthoc analyses revealed significant difference in the $\mathrm{HbO}(P=0.01 ; d=2.46)$ between easy $(0.07 \pm 0.01 \mu \mathrm{mol})$ and difficult $(0.15 \pm 0.01 \mu \mathrm{mol})$ conditions. Similarly, there was also a significant difference $(P=0.01 ; d=2.41)$ between

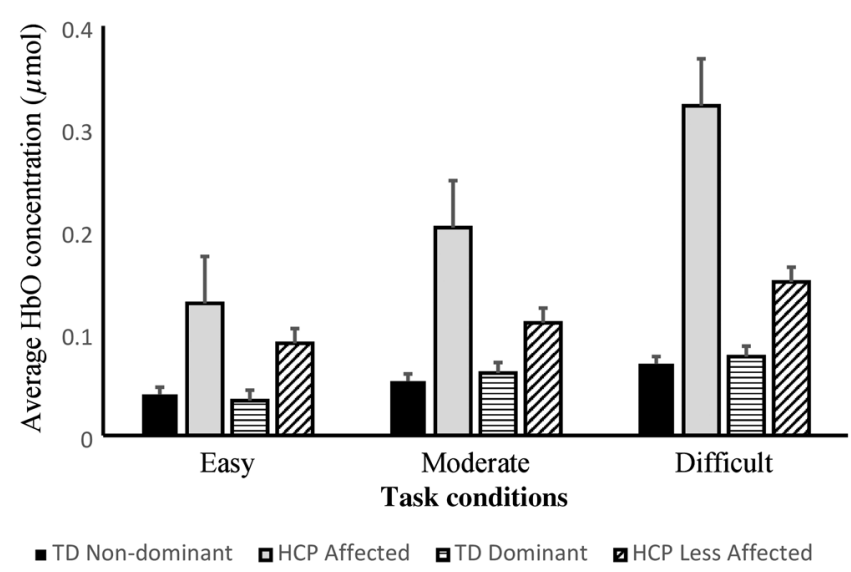

Fig. 3 Comparison of arm-specific concentration of oxygenated hemoglobin $(\mathrm{HbO})$ between TD children and children with $\mathrm{HCP}$ while performing the easy, moderate, and difficult shape-matching conditions. The data represent the combined activity across the right and left hemispheres of the prefrontal cortices. The concentration of $\mathrm{HbO}$ appeared to be highest for the difficult condition followed by moderate and easy condition. Furthermore, it is apparent that the concentration of $\mathrm{HbO}$ in the prefrontal cortices was highest for the children with HCP for all of the respective conditions. Error bars are standard error of mean.
Table 3 Main and interaction effects for all outcomes. Only significant results are shown.

\begin{tabular}{llccc} 
Outcomes & & $F$ & $P$ value & Effect size \\
\hline HbO & Group & 62.03 & 0.001 & 3.12 \\
& Arm & 11.24 & 0.001 & 1.32 \\
& Group $\times$ arm & 13.28 & 0.001 & 1.44 \\
& Condition & 10.97 & 0.001 & 1.31 \\
Task performance & Group & 70.95 & 0.001 & 11.1 \\
& Task condition & 26.51 & 0.001 & 5.3 \\
& Arm & 12.12 & 0.001 & 3.6 \\
& Group $\times$ arm & 8.65 & 0.004 & 9.5 \\
RT & Group & 33.58 & 0.001 & 6.5 \\
Task errors & Group & 47.93 & 0.001 & 8.4 \\
& Arm & 7.20 & 0.01 & 2.6 \\
& Group & 29.82 & 0.001 & 6.4 \\
NHPT & Group & 8.03 & 0.007 & 2.5 \\
& & 6.81 & 0.01 & 6.2 \\
& Group & 21.36 & 0.001 & 5.9 \\
\hline
\end{tabular}

moderate $(0.10 \pm 0.01 \mu \mathrm{mol})$ and difficult $(0.15 \pm 0.01 \mu \mathrm{mol})$ conditions. Altogether, these results indicate that the complexity of the shape-matching task promoted the expected changes in the $\mathrm{HbO}$ within the PFC.

We found a significant group main effect $(P=0.001$; $d=3.12)$ indicating that the children with $\mathrm{HCP}(0.17 \pm$ $0.01 \mu \mathrm{mol})$ had greater $\mathrm{HbO}$ than the TD children $(0.06 \pm$ $0.006 \mu \mathrm{mol}$ ) overall when completing the respective shapematching task conditions. There also was a significant group by condition interaction ( $P=0.025 ; d=0.76$ ). Posthoc analyses revealed a significant difference in $\mathrm{HbO}$ between the TD children and children with HCP during the easy $(P=0.022$; $d=2.86)$, moderate $(P=0.006 ; d=3.92)$, and difficult $(P=0.001 ; d=5.69)$ task conditions (Fig. 4). Hence, indicating that the children with $\mathrm{HCP}$ had a larger concentration of $\mathrm{HbO}$ while performing the respective task conditions when the data from the respective arms were combined. Despite this finding, the group $\times$ arm $\times$ condition interaction term was not significant $(P=0.11)$.

There was also a significant arm main effect $(P=0.001$; $d=1.32$ ) with the greater $\mathrm{HbO}$ when children performed the task with the nondominant/affected arm $(0.13 \pm 0.01 \mu \mathrm{mol})$ than the dominant/less affected arm $(0.08 \pm 0.008 \mu \mathrm{mol})$. There was a significant group by arm interaction $(P=0.001 ; d=$ 1.44). Posthoc analyses revealed a significant difference $(P=$ $0.001 ; d=2.56)$ in $\mathrm{HbO}$ when the task was performed with the affected arm $(0.22 \pm 0.03 \mu \mathrm{mol})$ of children with HCP and the nondominant arm of TD children $(0.05 \pm 0.008 \mu \mathrm{mol})$. Similarly, there was a significant $(P=0.001 ; d=0.67)$ 


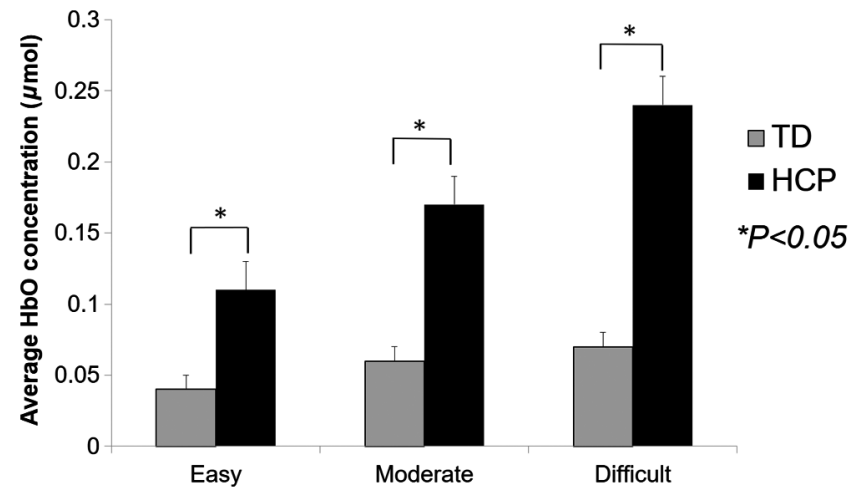

Fig. 4 Comparison of concentration of the average oxygenated hemoglobin $(\mathrm{HbO})$ between TD children and children with $\mathrm{HCP}$ while performing the easy, moderate, and difficult shape-matching conditions. These results are from the combined data from the respective arms and right/left hemispheres of the prefrontal cortices. The concentration of $\mathrm{HbO}$ was highest for the difficult condition followed by moderate and easy condition. Furthermore, it is apparent that the concentration of $\mathrm{HbO}$ in the prefrontal cortices was highest for the children with HCP for all of the respective conditions. Error bars are standard error of mean. * denotes $P \leq 0.05$.

difference in $\mathrm{HbO}$ concentration when the task was performed with the less affected arm $(0.12 \pm 0.01 \mu \mathrm{mol})$ of children with HCP and the dominant arm $(0.06 \pm 0.009 \mu \mathrm{mol})$ of TD children. There was also a significant difference $(P=0.001$; $d=1.62)$ in $\mathrm{HbO}$ between the affected $(0.22 \pm 0.03 \mu \mathrm{mol})$ and the less affected $(0.12 \pm 0.01 \mu \mathrm{mol})$ arm of children with HCP.

There was no significant main effect for the respective sides of the fNIRS probe $(P=0.91)$. Hence, indicating that the activity in the respective hemispheres was equivocal.

\subsection{Task Performance}

There was a significant group main effect $(P=0.001$; $d=11.1$ ) for the number of shapes matched, with TD children matching more shapes ( $8.02 \pm 0.2$ shapes) than the children with HCP $(5.2 \pm 0.3$ shapes $)$. There was a significant condition main effect $(P=0.001 ; d=5.3)$. Posthoc analyses indicated that children matched a greater number of shapes in easy $(8.13 \pm 0.3$ shapes $)$ than moderate $(6.53 \pm 0.3$ shapes; $P=0.001 ; \quad d=5.3)$ and difficult $(5.10 \pm 0.3$ shapes; $P=0.001 ; d=10)$ conditions. There was a significant arm main effect $(P=0.001 ; d=3.6)$, indicating that the number of shapes matched by the dominant/less affected arm (7.28 \pm 0.3 shapes) was greater than what was completed by the nondominant/affected arm (6.21 \pm 0.3 shapes). There also was a significant group by arm interaction $(P=0.004)$. Posthoc analyses revealed significant difference $(P=0.001$; $d=9.5$ ) in the number of shapes matched by the affected arm of children with HCP $(4.1 \pm 0.4$ shapes) was less than what was matched by the nondominant arm of TD children ( $7.9 \pm 0.4$ shapes). Similarly, the number of shapes matched with the less affected arm of children with HCP $(6.3 \pm 0.4$ shapes) was significantly less $(P=0.004 ; d=4.5)$ than the number completed by the dominant arm of TD children ( $8.1 \pm 0.4$ shapes). Lastly, for the children with HCP the number of shapes matched by the affected arm ( $4.1 \pm 0.4$ shapes) was significantly $(P=0.001 ; d=5.5)$ less than the number of shapes completed for the less affected arm ( $6.3 \pm 0.4$ shapes). None of the other interaction terms were significant $(P>0.05)$.

\subsection{Reaction Time}

There was a significant group main effect for RT $(P=0.001$; $d=6.5)$, indicating that overall the TD $(0.9 \pm 0.05 \mathrm{~s})$ had a faster RT than the children with HCP $(2.31 \pm 0.3 \mathrm{~s})$. None of the other main effects or interaction terms were significant $(P>0.05)$.

\subsection{Task Errors}

There was a significant group main effect $(P=0.001 ; d=8.4)$, indicating that the TD children $(1.4 \pm 0.2$ errors $)$ had fewer errors during the shape-matching tasks than children with HCP (4.6 \pm 0.5 errors). There also was a significant arm main effect $(P=0.01 ; d=2.6)$, signifying that there were fewer task errors for the dominant/less affected $(2.26 \pm 0.39$ errors) and the nondominant/affected arms ( $3.44 \pm 0.52$ errors). None of the other main effects or interaction terms were significant $(P>0.05)$.

\subsection{Nine-Hole Peg Test}

There was a significant group main effect $(P=0.001 ; d=6.4)$, showing that the TD children $(41.03 \pm 1.9 \mathrm{~s})$ were faster at completing the NHPT than the children with HCP $(96.1 \pm 12.0 \mathrm{~s})$. There also was a significant arm main effect $(P=0.007 ; d=2.5)$ indicating that the NHPT was completed faster with the dominant/less affected arm $(53.46 \pm 5.28 \mathrm{~s})$ than the nondominant/affected arm $(78.44 \pm 11.64 \mathrm{~s})$. There was a significant group $\times$ arm interaction $(P=0.01)$. Posthoc analyses revealed children with HCP were significantly $(P=0.001$; $d=6.2$ ) slower at the NHPT when they used affected arm $(130.5 \pm 19.8 \mathrm{~s})$ compared with when the TD children used their nondominant arm $(42.6 \pm 2.6 \mathrm{~s})$. Similarly, the children with HCP were significantly slower $(P=0.004 ; d=4.3)$ when they used less affected arm (68.8 $\pm 9.2 \mathrm{~s})$ compared with when the TD children used their dominant arm $(39.5 \pm 2.9 \mathrm{~s})$. In addition, the children with HCP performed the NHPT significantly $(P=0.03 ; d=4.0)$ slower with their affected arm compared with their less affected arm.

\subsection{Box and Block Test}

There was significant group main effect $(P=0.001 ; d=5.9)$ indicating that overall the TD children $(33.03 \pm 1.3$ blocks) moved more blocks than and children with HCP $(20.5 \pm 2.7$ blocks). There was no significant arm main effect $(P=0.11)$ or interaction $(P=0.06)$.

\subsection{Correlation Analyses}

There was a positive correlation between the concentration of $\mathrm{HbO}$ and the RT for the easy $(r=0.25, P=0.06)$, moderate $(r=0.43, P=0.04)$, and difficult $(r=0.72, P=0.01)$ complexity levels. Overall, these relationships implied that a greater concentration of $\mathrm{HbO}$ in the PFC during the sequential shapematching task was associated with a slower RT.

There was a positive correlation between the concentration of $\mathrm{HbO}$ easy $(r=0.34, P=0.014)$, moderate $(r=0.39$, $P=0.023$ ), and difficult ( $r=0.43, P=0.012$ ) complexity levels and the performance on the NHPT. Hence, the children that 


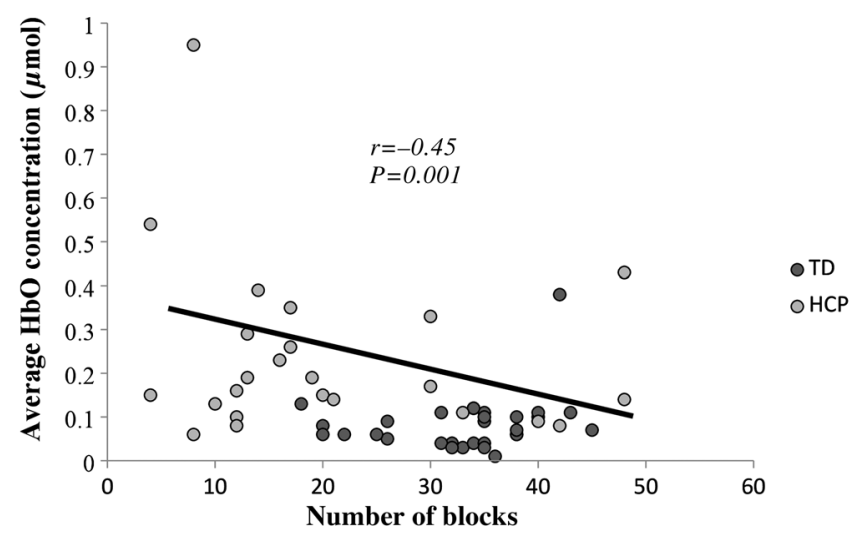

Fig. 5 Scatter plot of the PFC activation while performing the difficult shape-matching condition and BBT. Abscissa represents number of blocks that children completed and the ordinate represents average oxygenated hemoglobin $(\mathrm{HbO})$ concentration. There was a moderate negative correlation $(r=-0.45, P=0.001)$, which suggests that children that moved fewer blocks tended to have greater concentration of $\mathrm{HbO}$ in the PFC during the difficult shape-matching condition.

performed slower on the NHPT also tended to have a greater concentration of $\mathrm{HbO}$ in the $\mathrm{PFC}$ during the sequential shape-matching tasks.

Lastly, there was a moderate negative correlation between the concentration of $\mathrm{HbO}$ during the easy $(r=-0.35, P=0.010)$, moderate $(r=-0.38 ; P=0.005)$, and difficult $(r=-0.45$, $P=0.001$; Fig. 5) complexity levels and performance on the BBT. This result suggests that children that moved fewer blocks tended to have greater concentration of $\mathrm{HbO}$ in the PFC during the sequential shape-matching tasks.

\section{Discussion}

This study showed that an increase in the complexity of the shape-matching task resulted parallel increases in the PFC activation. More importantly, our results identified that children with HCP have higher PFC activation while performing a shape-matching motor task with their affected upper extremities across the various shape-matching complexity levels. Interestingly, the greater PFC activation was also seen when the children with HCP performed the shape-matching motor task with the less affected hand. The heightened activity seen within the PFC was related to a slower RT and poorer performance on the NHPT and BBT. Taken together, these results suggest that the atypical motor actions seen in children with HCP may be partially related to the greater demands placed on the PFC when planning and executing a goal-directed movement with the upper extremities.

The increased activation seen in the PFC while performing the shape-matching task with the affected as well as less affected arm may indicate that children with HCP have difficulty in simultaneously processing the cognitive and motor demands of the task. For example, the children had to simultaneously perform a series of motor actions with the affected arm while selecting an object and identify where it belonged on the template. Hence, it is possible that heightened PFC activation may partially reflect competition between the motor and cognitive resources that were necessary for completing the task demands. This notion corroborates with a prior investigation that has suggested that children with HCP have deficits in cognitive processing. ${ }^{52}$ This may indicate that the brain insults seen in children with
HCP are not limited to sensorimotor areas only, but rather they have a cascading effect on the other cortical areas.

The children with HCP had higher PFC activation in both hemispheres. As opposed to motor cortex, the activation in the PFC was not laterality specific since there was no differential activation within the right and left PFCs while performing the task with the affected or the less affected arm. Greater bilateral activation within the PFC could be due to the recruitment of homologous resources across both PFC due to greater demands imposed while planning and executing the complex shapematching task. Or alternatively, activation in both hemispheres may be caused by the concomitant activity of various separable cognitive processes that differentially involve both the left and right PFCs while planning the complex task. ${ }^{53,54}$ These study results corroborate with the other neuroimaging studies that demonstrated bilateral PFC and dorsolateral PFC activation while planning and executing a motor task. ${ }^{55-62}$

The children with HCP matched a fewer number of shapes, had longer RT, and more shape matching errors compared with the TD children. Altogether these behavioral results indicate that the shape-matching task was more difficult for the children with HCP. It could be argued that the ability to match a fewer number of shapes potentially originate from faults in the musculoskeletal machinery (i.e., spasticity, weakness, and joint contractures). Although plausible, this argument is questionable because there was a correlation between the motor performance on the shapematching task and the activity within the PFC, and the deficits remained even when the children performed the task with their less affected arm. These correlations imply that the musculoskeletal impairments are not solely responsible for reduced motor performance; rather, deficient cognitive processing may also underlie the uncharacteristic motor performance.

The motor impairments seen in the children with HCP while performing the shape matching tasks were further confirmed by the outcomes of the BBT and NHPT. Children with HCP completed fewer blocks during the BBT and took longer time to complete the NHPT. Thus, the children with HCP had reduced manual speed and dexterity bilaterally, which corresponds to the finding that the children with HCP matched fewer numbers of shapes and had increased shape-matching errors. The results from the correlation analysis implied that the reduced motor performance on these clinical tests might also be linked with the greater PFC activation. These correlations provide further fuel for the notion that a higher PFC activation might indicate a heightened demand on the available cortical resources available for children with HCP.

One of the major limitations of the present study is that a limited number of optodes were used, and our analysis was restricted to the PFC. Moreover, the other areas associated with action planning such as the fronto-parietal cortical areas, basal ganglia, and cerebellum were not evaluated simultaneously. Potentially, deficits in these cortical and subcortical areas may have a larger influence on the action-planning deficits seen in children with HCP. Secondly, we did not have electromyographic or kinesiological data to measure the motor impairments that may reside in musculoskeletal system. Therefore, our study results are inadequate in partitioning whether the uncharacteristic motor performance seen in children with $\mathrm{HCP}$ is due to impaired musculoskeletal machinery and/or aberrant cortical processes. Lastly, the data processing techniques employed in this investigation assumed that the physiological noise embedded within the measured cortical hemodynamic response 
was sufficiently removed. Our analysis may have not accounted for the overlapping frequency bands between the blood pressure, respirator signal, and the cerebral hemodynamic response. Several postprocessing methods and concurrent external measurement techniques (i.e., respiration, blood pressure) have been proposed to better extract the cortical hemodynamic response, but currently there is not a clear standard that has emerged for eliminating the physiological noise that is often seen in fNIRS. ${ }^{63,64}$ Overall, these noted limitations should be taken into consideration in future studies that are directed at understanding the action-planning deficits in children with HCP.

\section{Conclusion}

Our results show that children with HCP have increased activation in the PFC while performing a shape-matching motor task with their affected and less affected upper extremities. This suggests that the children with HCP may utilize greater cognitive and attentional resources to plan and execute their goal-directed actions. In addition, our results indicate that the children with HCP have slower RTs and generate more errors during their goal-directed actions, even in the less affected extremity. These parallel results imply that the motor performance problems seen in children with HCP could be due to an underlying cognitive processing and/or action-planning deficits associated with the PFC. Therefore, therapeutic approaches that improve the motor actions of children with HCP might also result in corollary changes in their cognitive processing. This unique perspective should be challenged by future studies because it may identify unforeseen connections between the motor impairments seen in children with HCP and their cognitive abilities.

\section{Disclosures}

The authors have stated that they had no interests that might be perceived as posing a conflict or bias.

\section{Acknowledgments}

The World Bank-Margaret McNamara Fund (MMMF) Education Grant, American Association of University Women (AAUW) International Doctoral Fellowship, North American Society for the Psychology of Sports and Physical Activity (NASPSPA), the National Science Foundation (NSF 1539067) and the National Institutes of Health (1R011HD086245) provided partial funding for this investigation. We would like to acknowledge Dr. Hasan Ayaz for his consultation on the use the fNIRS system, and Brenda Davies and Michael Trevarrow for their assistance with the data collections.

\section{References}

1. L. Sakzewski, J. Ziviani, and R. Boyd, "Systematic review and metaanalysis of therapeutic management of upper-limb dysfunction in children with congenital hemiplegia," Pediatrics 123(6), e1111-e1122 (2009).

2. R. Boyd, M. Morris, and H. Graham, "Management of upper limb dysfunction in children with cerebral palsy: a systematic review," Eur. J. Neurol. 8(s5), 150-166 (2001).

3. B. Steenbergen and A. Gordon, "Activity limitation in hemiplegic cerebral palsy: evidence for disorders in motor planning," Dev. Med. Child Neurol. 48(09), 780-783 (2006).

4. M. Kurz et al., "Neurophysiological abnormalities in the sensorimotor cortices during the motor planning and movement execution stages of children with cerebral palsy," Dev. Med. Child Neurol. 56(11), 10721077 (2014).
5. C. Kaller et al., "Dissociable contributions of left and right prefrontal cortex in planning," Cereb. Cortex 21(2), 307-317 (2011).

6. S. Glover, "Separate visual representations in the planning and control of action," Behav. Brain Sci. 27, 3-24 (2004).

7. S. Glover, M. Wall, and A. Smith, "Distinct cortical networks support the planning and online control of reaching-to-grasp in humans," Eur. J. Neurosci. 35(6), 909-915 (2012).

8. M. Mutsaarts, B. Steenbergen, and H. Bekkering, "Anticipatory planning deficits and task context effects in hemiparetic cerebral palsy," Exp. Brain Res. 172(2), 151-162 (2006).

9. B. Steenbergen, R. Meulenbroek, and D. Rosenbaum, "Constraints on grip selection in hemiparetic cerebral palsy: effects of lesional side, end-point accuracy, and context," Cognit. Brain Res. 19(2), 145-159 (2004)

10. S. Duff and A. Gordon, "Learning of grasp control in children with hemiplegic cerebral palsy," Dev. Med. Child Neurol. 45(11), 746757 (2003).

11. B. Steenbergen and J. Van Der Kamp, "Control of prehension in hemiparetic cerebral palsy: similarities and differences between the ipsi-and contra-lesional sides of the body," Dev. Med. Child Neurol. 46(05), 325-332 (2004).

12. M. Mutsaarts, B. Steenbergen, and H. Bekkering, "Anticipatory planning of movement sequences in hemiparetic cerebral palsy," Mot. Control 9(4), 439-458 (2005).

13. C. Sahyoun et al., "Towards an understanding of gait control: brain activation during the anticipation, preparation and execution of foot movements," NeuroImage 21(2), 568-575 (2004).

14. J. Gallivan et al., "Where one hand meets the other: limb-specific and action-dependent movement plans decoded from preparatory signals in single human frontoparietal brain areas," J. Neurosci. 33(5), 1991-2008 (2013).

15. M. Kurz et al., "Developmental trajectory of beta cortical oscillatory activity during a knee motor task," Brain Topogr. 29(6), 824-833 (2016).

16. A. Luft et al., "Comparing brain activation associated with isolated upper and lower limb movement across corresponding joints," Hum. Brain Mapp. 17(2), 131-140 (2002).

17. B. MacIntosh et al., "Optimizing the experimental design for ankle dorsiflexion fMRI," NeuroImage 22(4), 1619-1627 (2004).

18. E. Kapreli et al., "Lateralization of brain activity during lower limb joint movement. An fMRI study," NeuroImage 32(4), 1709-1721 (2006).

19. S. Beurze et al., "Integration of target and effector information in the human brain during reach planning," J. Neurophys. 97(1), 188-199 (2007).

20. J. Gallivan et al., "Decoding action intentions from preparatory brain activity in human parieto-frontal networks," J. Neurosci. 31(26), 9599-9610 (2011).

21. K. Valyear and S. Frey, "Human posterior parietal cortex mediates handspecific planning," NeuroImage 114, 226-238 (2015).

22. A. Owen, "Cognitive planning in humans: new insights from the Tower of London (TOL) task," in The Cognitive Psychology of Planning, pp. 135-151, Taylor \& Francis, Psychology Press, United Kingdom (2005).

23. S. Witt, A. Laird, and M. Meyerand, "Functional neuroimaging correlates of finger-tapping task variations: an ALE meta-analysis," Neurolmage 42(1), 343-356 (2008).

24. L. Carr et al., "Patterns of central motor reorganization in hemiplegic cerebral palsy," Brain 116(Pt 5), 1223-1247 (1993).

25. M. Staudt et al., "Two types of ipsilateral reorganization in congenital hemiparesis: a TMS and fMRI study," Brain 125(Pt 10), 2222-2237 (2002).

26. Y. Maegaki, "Central motor reorganization in cerebral palsy patients with bilateral cerebral lesions," Pediatr. Res. 45, 559-567 (1999).

27. Y. Vandermeeren et al., "Long-latency motor evoked potentials in congenital hemiplegia," Clin. Neurophysiol. 114(10), 1808-1818 (2003).

28. L. Holmstrom, "Hand function in relation to brain lesions and corticomotor-projection pattern in children with unilateral cerebral palsy," Dev. Med. Child Neurol. 52(2), 145-152 (2010).

29. A. Hoon et al., "Sensory and motor deficits in children with cerebral palsy born preterm correlate with diffusion tensor imaging abnormalities in thalamocortical pathways," Dev. Med. Child. Neurol. 51(9), 697-704 (2009). 
30. Y. Vandermeeren et al., "Functional reorganization of brain in children affected with congenital hemiplegia: fMRI study," NeuroImage 20(1), 289-301 (2003).

31. A. Guzzetta et al., "Reorganisation of the somatosensory system after early brain damage," Clin. Neurophysiol. 118(5), 1110-1121 (2007).

32. M. Wilke et al., "Somatosensory system in two types of motor reorganization in congenital hemiparesis: topography and function," Hum. Brain Mapp. 30(3), 776-788 (2009).

33. K. Manning et al., "Neuroplastic sensorimotor resting state network reorganization in children with hemiplegic cerebral palsy treated with constraint-induced movement therapy," J. Child Neurol. 31(2), 220-226 (2016)

34. M. Walther et al., "Motor cortex plasticity in ischemic perinatal stroke: a transcranial magnetic stimulation and functional MRI study," Pediatr. Neurol. 41(3), 171-178 (2009).

35. C. Morris et al., "Reliability of the manual ability classification system for children with cerebral palsy," Dev. Med. Child. Neurol. 48(12), 950953 (2006).

36. L. Krumlinde-Sundholm et al., "The assisting hand assessment: current evidence of validity, reliability, and responsiveness to change," Dev. Med. Child. Neurol. 49(4), 259-264 (2007).

37. L. Krumlinde-Sundholm, "Reporting outcomes of the assisting hand assessment: what scale should be used?" Dev. Med. Child Neurol. 54(9), 807-808 (2012).

38. J. Poole et al., "Measuring dexterity in children using the nine-hole peg test," J. Hand Ther. 18(3), 348-351 (2005).

39. M. Jongbloed-Pereboom, M. Nijhuis-van der Sanden, and B. Steenbergen, "Norm scores of the box and block test for children ages 3-10 years," Am. J. Occup. Ther. 67(3), 312-318 (2013).

40. R. Oldfield, "The assessment and analysis of handedness: the Edinburgh inventory," Neuropsychologia 9(1), 97-113 (1971).

41. D. Boas, A. Dale, and M. Franceschini, "Diffuse optical imaging of brain activation: approaches to optimizing image sensitivity, resolution, and accuracy," Neurolmage 23, S275-S288 (2004).

42. H. Obrig and A. Villringer, "Beyond the visible-imaging the human brain with light," J. Cereb. Blood Flow Metab. 23(1), 1-18 (2003).

43. Y. Fukui, Y. Ajicki, and E. Okada, "Monte Carlo prediction of nearinfrared light propagation in realistic adult and neonatal head models," Appl. Opt. 42, 2881-2887 (2003).

44. E. Okada and D. T. Delpy, "Near-infrared light propagation in an adulat head model. II. Effect of superficial tissue thickness on the sensitivity of the near-infrared spectroscopy signal," Appl. Opt. 42, 2915-2922 (2003).

45. E. Okada and D. T. Delpy, "Near-infrared light propagation in an adult head model. I. Modeling of low-level scattering in the cerebrospinal fluid layer," Appl. Opt. 42, 2906-2914 (2003).

46. H. Ayaz, "Functional near infrared spectroscopy based brain computer interface," PhD Thesis, Drexel University, Philadelphia, Pennsylvania (2010).

47. R. Gentili et al., "Functional near-infrared spectroscopy-based correlates of prefrontal cortical dynamics during a cognitive-motor executive adaptation task," Front. Hum. Neurosci. 7, 1-13 (2013).

48. H. Ayaz et al., "Using mazesuite and functional near infrared spectroscopy to study learning in spatial navigation," J. Visualized Exp. 56, 3443 (2011).

49. M. Izzetoglu et al., "Functional brain imaging using near-infrared technology," IEEE Eng. Med. Biol. Mag. 26(4), 38-46 (2007).

50. M. Suzuki et al., "Prefrontal and premotor cortices are involved in adapting walking and running speed on the treadmill: an optical imaging study," NeuroImage 23(3), 1020-1026 (2004).

51. J. Cohen, Statistical Power Analysis for the Behavioral Sciences, 2nd ed., pp. 20-26, Lawrence Earlbaum Associates, Hillsdale, New Jersey (1988).

52. K. Murias et al., "A review of cognitive outcomes in children following perinatal stroke," Dev. Neuropsychol. 39(2), 131-157 (2014).
53. H. Ayaz et al., "Optical brain monitoring for operator training and mental workload assessment," NeuroImage 59(1), 36-47 (2012).

54. K. Izzetoglu et al., "Functional optical brain imaging using near-infrared during cognitive tasks," Int. J. Hum. Comput. Interact. 17(2), 211-227 (2004).

55. G. Ward and R. Morris, "Introduction to the psychology of planning," in The Cognitive Psychology of Planning, pp. 1-34, Taylor \& Francis, Psychology Press, United Kingdom (2005).

56. E. Koechlin et al., "Dissociating the role of the medial and lateral anterior prefrontal cortex in human planning," Proc. Natl. Acad. Sci. U. S. A. 97(13), 7651-7656 (2000).

57. E. Koechlin et al., "The role of the anterior prefrontal cortex in human cognition," Nature 399(6732), 148-151 (1999).

58. S. Baker et al., "Neural systems engaged by planning: a PET study of the Tower of London task," Neuropsychologia 34(6), 515-526 (1996).

59. A. Dagher et al., "Mapping the network for planning: a correlational PET activation study with the Tower of London task," Brain 122(10), 1973-1987 (1999).

60. R. Lazeron et al., "Visualizing brain activation during planning: the Tower of London test adapted for functional MR imaging," Am. J. Neuroradiol. 21(8), 1407-1414 (2000).

61. U. Chaudhary et al., "Motor response investigation in individuals with cerebral palsy using near infrared spectroscopy: pilot study," Appl. Opt. 53(3), 503-510 (2014).

62. Y. Moriguchi and K. Hiraki, "Prefrontal cortex and executive function in young children: a review of NIRS studies," Front. Hum. Neurosci. 7, 867 (2013).

63. E. Smith et al., "Prefrontal activation during executive tasks emerges over early childhood: evidence from functional near infrared spectroscopy," Dev. Neuropsychol. 42, 253-264 (2017).

64. Y. Moriguchi and K. Hiraki, "Longitudinal development of prefrontal function during early childhood," Dev. Cognit. Neurosci. 1(2), 153-162 (2011).

65. T. J. Huppert et al., "HomER: a review of time-series analysis methods for near-infrared spectroscopy of the brain," Appl. Opt. 48(10), D280D298 (2009).

66. N. Naseer and K. S. Hong, "fNIRS-based brain-computer interfaces: a review," Front. Hum. Neurosci. 9, 3 (2006).

Swati M. Surkar received her doctorate from the University of Nebraska Medical Center's Munroe-Meyer Institute and is currently a postdoctoral research associate at the Neurorehabilitation Research Lab, Washington University School of Medicine. Her research focuses on developing interventions for children and adults with neurological disorders, motor planning, motor learning, and brain imaging.

Rashelle M. Hoffman is a physical therapist and a Geriatric Certified Specialist. Currently, she is a PhD student at the University of Nebraska Medical Center's Munroe-Meyer Institute. Her research focuses on the motor actions and sensory processing of adults with cerebral palsy.

Regina Harbourne is an associate professor in the Department of Physical Therapy at Duquesne University. Her clinical specialty area is pediatrics. She directs research in the Infant Development Lab. Her research focuses on early postural control development, efficacy of early intervention, motor learning, and variability and complexity of motor control.

Max J. Kurz is an associate professor in the Munroe-Meyer Institute's Department of Physical Therapy at the University of Nebraska Medical Center. His research is directed at developing new therapeutic strategies that will empower children with cerebral palsy to learn new motor skills. 